\title{
Association of glycemic profiles with whole blood polyamine among middle-aged Japanese men: colorimetric assay using oat and barley seedling polyamine oxidase
}

\author{
Takaaki Kondo $\cdot$ Kanami Yamamoto $\cdot$ \\ Akiko Kimata · Jun Ueyama · Yoko Hori · \\ Kenji Takagi
}

Received: 22 May 2007 / Accepted: 1 August 2007/Published online: 11 December 2007

(C) The Japanese Society for Hygiene 2008

\begin{abstract}
Objectives Polyamines have long been known to have an insulin-like action, but their antiglycating effect has only recently attracted the attention of researchers. The aim of our investigation was to determine the whole blood polyamine concentration in a healthy population in order to examine its relationship with glycemic profiles.

Methods The study cohort comprised 622 men aged 4059 who participated in a health checkup program conducted in 1997, when they underwent measurements of fasting plasma glucose (FPG), insulin (FPI), and fructosamine as glycemic indices. Colorimetric assay methods using oat and barley seedling polyamine oxidase were used to determine total polyamine (spermidine + spermine) and spermine concentrations in the whole blood, respectively. Polyamine concentrations adjusted for hemoglobin were quartiled for the analysis of covariance to assess the association with glycemic indices.

Results A significant association was demonstrated between the FPG and total polyamine concentrations. In the trend test, FPG and fructosamine levels increased in accordance with the shift of quartiles of total polyamine
\end{abstract}

T. Kondo $\cdot$ K. Yamamoto $\cdot$ A. Kimata $\cdot$ J. Ueyama $\cdot$ K. Takagi Program in Radiology and Medical Laboratory Sciences, Nagoya University Graduate School of Medicine, Nagoya, Japan

Y. Hori

Program in Nursing, Nagoya University Graduate School of Medicine, Nagoya, Japan

T. Kondo $(\bowtie)$

Nagoya University School of Health Sciences,

1-1-20 Daikominami, Higashi-ku, Nagoya 461-8673, Japan

e-mail: taka-ngy@umin.ac.jp concentrations from low to high. In contrast, the association between the spermine and glycemic indices was not statistically significant based on the test for difference of multivariate-adjusted means or trend for linearity.

Conclusions This is the first epidemiological study to reveal that the concentrations of blood polyamines are related with either FPG or fructosamine level in a healthy population. There may be some feedback mechanism for the elevation of circulating polyamines to quench the glycation reaction under hyperglycemic conditions. In addition, total polyamines, rather than spermine alone, seem to be a sensitive biomarker representing the antiglycation effect of polyamines.

Keywords Analysis of covariance - Glycation · Polyamine oxidase $\cdot$ Spermidine $\cdot$ Spermine

\section{Introduction}

Prolonged exposure to hyperglycemic conditions causes free amino groups of protein to be derivatized by reactive carbonyl groups [1], which is the underlying mechanism involved in the development of such long-term diabetic complications as microvascular and macrovascular disorders. Naturally occurring polyamines, including spermidine (Spd) and spermine (Spm), are aliphatic polycations found in every living cell in quantities varying from the high molecular to low millimolar [2]. Polyamines have important roles in a large number of relevant biochemical and physiological functions [3]. One of these, their antiglycation property, has recently been attracting the attention of researchers. In an in vitro experiment, Nakamura et al. [4] demonstrated a chemical inhibition of the formation of advanced glycation end products (AGEs) as a result of the 
interaction between the free amino groups and highly reactive carbonyl compounds.

Although polyamines have been observed at relatively high concentrations in a number of pathophysiological conditions [5-8], the etiological significance of polyamines circulating at a physiological concentration has received relatively little attention in terms of its association with the development of chronically degenerative illnesses, including diabetes mellitus in general human populations.

Several methodologies have been reported for the quantification of the polyamines in biological samples [9]; of these, high-performance liquid chromatography (HPLC) remains the technique of choice for the polyamine assay because of its high reproducibility, sensitivity, and suitability for full automation. Although HPLC methods have been improved, resulting in HPLC systems that are faster, more sensitive, with a better resolution, such systems involve the use of sophisticated equipment and are labor-intensive. In practical terms, this hinders the application of such HPLC methods in epidemiological research for the assay of many samples. We have recently developed a colorimetric assay method for polyamines in packed erythrocytes [10] using oat seedling polyamine oxidase (OSPO). Smith intensively investigated OSPO in terms of its purification technique and chemical properties, including optimum $\mathrm{pH}$ for stability, stoichiometry, and effect of inhibitors [11-13]. Previous studies have demonstrated that OSPO possesses an extremely high activity toward Spd and Spm but absolutely no activity toward putrescine $[14,15]$. Takagi et al. [10] confirmed our new assay method to be superior in terms of sensitivity, simplicity, and rapidity, and we have subsequently attempted to apply it in our epidemiological research on the association between the whole blood polyamine $(\mathrm{Spd}+\mathrm{Spm})$ concentration and a number of metabolic risk factors. Smith also characterized barley seedling polyamine oxidase (BSPO), which is particularly active with Spm, and explored its optimal assay conditions for Spm oxidation $[12,16]$. We utilized this specific property of BSPO for determining the whole blood Spm concentration by slightly modifying the OSPO-based polyamine quantification method.

Based on reports indicating the antiglycation effects of polyamines, some researchers have speculated on the involvement of circulating polyamines in controlling the levels of plasma glucose in healthy persons. One possibility is that a high plasma glucose level may induce a protective feedback mechanism of polyamines, thus resulting in the elevation of polyamine concentrations to inhibit glycation. Another possibility, based on experimental evidence showing the insulin-like effect of polyamines on lipoprotein lipase activity or glucose metabolism, is that a higher concentration of circulating polyamines may result in a lower glucose [17, 18]. To our knowledge, however, no research has been carried out on humans to date that confirms these twofold speculations. The purpose of this study, therefore, was to examine the relationship between the whole blood polyamine concentrations and some indices of glycemic profiles in a middle-aged, healthy, working male population.

\section{Material and methods}

\section{Study population}

This study used data from 1143 male employees of the local government, Aichi Prefecture, central Japan, who participated in a comprehensive health check-up program conducted in 1997. Women were excluded because of their relatively small number $(n=222)$ among the participants and suggested menstrual cycle-related changes in circulating Spm concentrations in healthy women [19]. Eligible subjects were aged 40-59 years, had completed a self-administered questionnaire sheets covering their present history of diseases and such lifestyle characteristics as smoking status, drinking habits, and leisure-time physical activity, and had undergone anthropometric measurements of height and weight for the calculation of body mass index (BMI, $\mathrm{kg} / \mathrm{m}^{2}$ ) as well as measurements of overnight fasting hemoglobin $(\mathrm{Hb}, \mathrm{g} / \mathrm{dl})$, plasma glucose (FPG, mmol/l), insulin (FPI, $\mu \mathrm{U} / \mathrm{ml}$ ), and fructosamine $(\mu \mathrm{mol} / \mathrm{l})$. A subsequent eligibility criterion limited participants to those individuals who gave written informed consent to using their residual blood samples, and the results of the biochemical analyses of that blood, for our research purpose. We thus obtained a study cohort consisting of 1076 men, among whom 622 individuals (57.8\%) were selected for the current study. This sample size was more than double the size of the study population in our preliminary study $(n=290)$, in which the statistical power was found to be somewhat inadequate to detect the relationship of blood polyamines with a number of biochemical markers. Simple random sampling by reference to random number tables was chosen to select the subjects without multi-staging or stratification based on the characteristics of the those examined in the checkup.

The health check-up was conducted in the Aichi Prefectural Center of Health Care, Nagoya, Japan throughout 1997, and residual whole blood samples were stored at $-80^{\circ} \mathrm{C}$ in a deep freezer until assay. The study protocol was approved by the Ethics Committee of the Nagoya University School of Medicine, Nagoya, Japan. 
Polyamine assay

The purification of enzymes to be used for determining the levels of polyamines was carried out according to methods reported for earlier studies, with slight modification [1113, 16]. Briefly, OSPO and BSPO were extracted from leaves of oat (Avena sativa) and barley (Hordeum vulgare) seedlings, respectively, which were both grown in darkness at approximately $25^{\circ} \mathrm{C}$ for 2 weeks. The homogenized dark-grown leaves were eluted in $\mathrm{NaCl}$, precipitated in acetone precipitation, re-dissolved in citrate, and dialyzed in the presence of cellulose phosphate against $\mathrm{NaCl}$-free citrate buffer for $18 \mathrm{~h}$. The enzyme fractions were recovered from the cellulose phosphate precipitate after ammonium sulfate fractionation, and the supernatant was purified by second 18-h dialysis against citrate buffer to remove excessive ammonium sulfate. The preparations of OSPO and BSPO were stored at $4^{\circ} \mathrm{C}$ and frozen at $-30^{\circ} \mathrm{C}$, respectively.

The principle underlying the polyamine assay methods we employed is that, in proportion to the amount of hydrogen peroxide that is formed by either the OSPO- or BSPO-catalyzed degradation of polyamines, $N$-(carboxymethylaminocarbonyl)-4,4'-bis(dimethlylamino)diphenylamine sodium salt (DA-64) is quantitatively oxidized in the presence of peroxide, resulting in production of $\mathrm{N}$-(4\{[4-(dimethylamino)phenyl]imino $\}$-2,5-cyclohexadien-1ylidene)- $N$-methylmethanaminium chloride (Bindschelder's Green), which was measured at $727 \mathrm{~nm}$ on a spectrophotometer (Fig. 1). To enable whole blood samples instead of packed erythrocytes to be assayed for Spd + Spm determination, we conducted a preliminary experiment to optimize the $\mathrm{pH}$. A similar experiment was carried out to optimize $\mathrm{pH}$ in BSPO-catalyzed polyamine oxidation for the whole blood Spm determination.

\section{Statistical analysis}

We used the FPG and FPI concentrations to evaluate the glycemic profiles with which the association of whole blood polyamines is statistically analyzed. We also adopted the homeostasis model of assessment-insulin resistance $($ HOMA-IR $=$ FPG $\times$ FPI/22.5) and fructosamine as an index of insulin resistance [20] and as an index of glycation of serum protein [21], respectively.

Because the majority of the circulating Spm and Spd are contained in red blood cells [22] and their concentrations in blood plasma are low (within the range of $\mathrm{pmol} / \mathrm{ml}$; [23]), the differences in plasma volume should be eliminated. For this purpose, we calculated the polyamine concentration corrected as a ratio of polyamine to $\mathrm{Hb}$. The subjects were then grouped into quartiles according to the adjusted polyamine concentrations, and the analysis of covariance (ANCOVA) was performed to estimate the least-squares means of each glycemic index for comparison across those quartiles. Multivariate adjustment was made for age and BMI (Model 1), and further adjustment was conducted for the factors of smoking status (current, former, never), drinking habits (none, light: daily alcohol consumption approximately less than $23 \mathrm{~g}$; moderate: $23-46 \mathrm{~g}$; heavy: $46 \mathrm{~g}$ or over), leisure-time physical activity (regularly active, somewhat active, not very active), and a present history of any treatment for hypertension, diabetes,
Fig. 1 Polyamine assay procedure (Nagoya, Japan 2006). TCA Trichloroacetic acid, $P I P E S$ piperazine- $N, N^{\prime}$-bis (2-ethanesulfonic acid), $O S P O$ oat seedling polyamine oxidase, $B S P O$ barley seedling polyamine oxidase, DA64 $\mathrm{N}$-(carboxymethylaminocarbonyl)-4,4'-bis(dimethlylamino)diphenylamine sodium salt, $P O D$ peroxidase

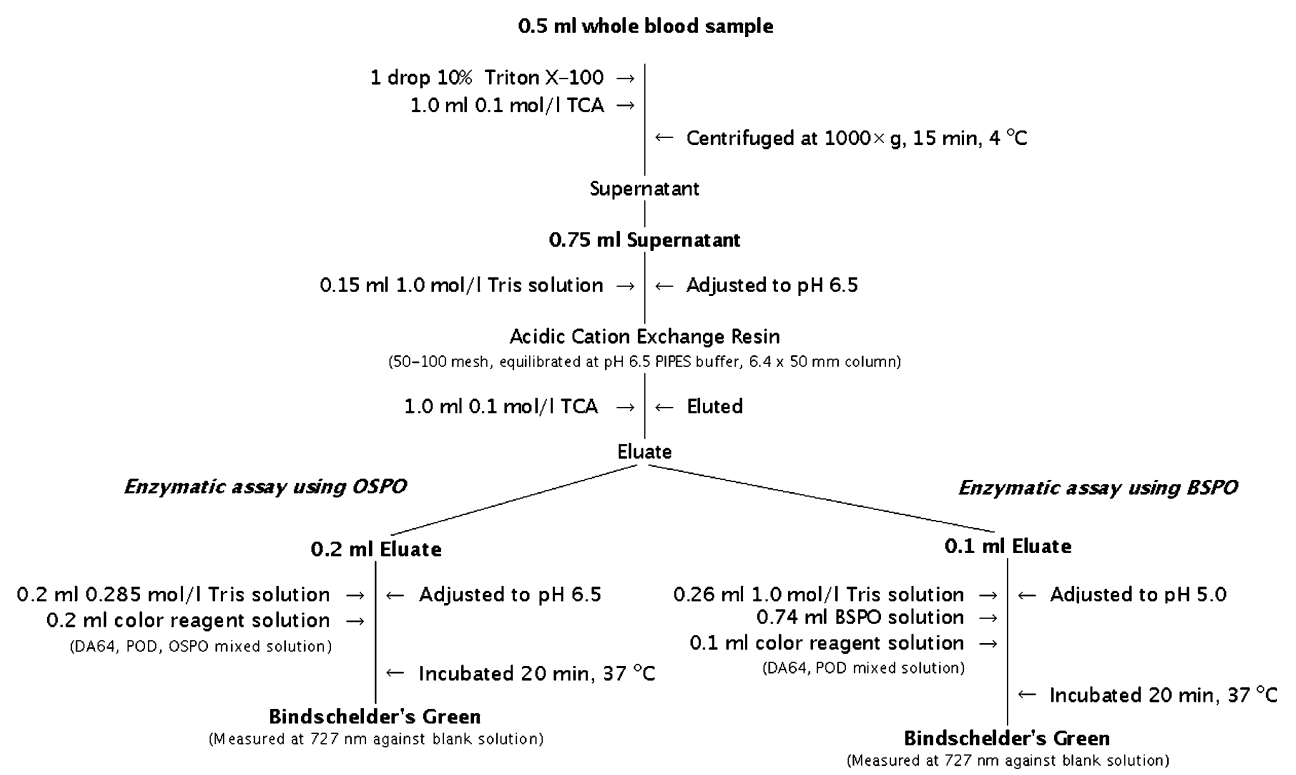


dyslipidemia, stroke, or hyperuricemia (Model 2). The classification method for the intensity of both drinking habits and leisure-time physical activity has been thoroughly described in a previous paper [24]. To test the linear trend for each glycemic index according to the polyamine concentrations, we used a linear contrast on the assumption that the polyamine quartiles were equally spaced from low to high. Data management and analysis were conducted using SPSS for Windows ver. 11.0 (SPSS, Chicago, IL). In all statistical analyses, two-sided $P$ values $<0.05$ were considered to be statistically significant.

\section{Results}

The average age of the subjects was $50.6 \pm 6.3$ years (mean $\pm \mathrm{SD}$ ), and their mean BMI was $23.4 \pm 2.6 \mathrm{~kg} / \mathrm{m}^{2}$, with $26.5 \%$ of the population having a $\mathrm{BMI} \geq 25$. The respective means of FPG, FPI, HOMA-IR, and fructosamine concentrations were $5.76 \pm 1.25 \mathrm{mmol} / \mathrm{l}, 5.94 \pm 4.82 \mu \mathrm{U} /$ $\mathrm{ml}, 1.53 \pm 1.22 \mathrm{mmol} / \mathrm{l}$, and $255.7 \pm 36.9 \mu \mathrm{mol} / \mathrm{l}$. The uncorrected concentrations of whole blood polyamines were $15.7 \pm 7.9 \mathrm{nmol} / \mathrm{ml}$ for Spd $+\mathrm{Spm}$ and $7.8 \pm 5.6 \mathrm{nmol} / \mathrm{ml}$ for $\mathrm{Spm}$ ): these were converted after $\mathrm{Hb}$ correction to $0.105 \pm 0.059$ and $0.052 \pm 0.038 \mu \mathrm{mol} / \mathrm{g}$, respectively. Current smokers accounted for $35 \%$ of the subjects, and moderate to heavy drinkers for $47 \%$. Some $17.5 \%$ of the participants were under treatment for one or more of the following: hypertension, diabetes, dyslipidemia, stroke, heart disease, or hyperuricemia.

Statistically significant differences in means of age, FPG and HOMA-IR were indicated according to the quartile of Spd + Spm (Table 1), whereas no significant associations of either BMI, FPI, or fructosamine with Spd + Spm were found. Neither smoking status, drinking habit, nor leisuretime physical activity was related to the quartile of Spd + $\mathrm{Spm}$, and a present history of any chronic degenerative disease under treatment was unrelated to the Spd $+\mathrm{Spm}$ concentration. In relation to the quartiled Spm concentration, means of age remained statistically significant, but those of the other measurements, including BMI, FPG, FPI, HOMA-IR, and fructosamine no longer differed at a statistically significant level between the quartiles (Table 2). Moreover, the contingency tables for the association of either smoking status, drinking habit, leisure-time physical activity, or a present history of any chronic degenerative disease with the quartile of Spm yielded no statistically significant relationship.

With respect to the relationship between the means of FPG and quartiles of Spd + Spm concentrations (Table 3), age- and BMI-adjustment yielded a highly significant association (Model 1), which was essentially unchanged after the additional adjustment was made for the effects of smoking status, drinking habit, leisure-time physical activity, and present history of chronic disease (Model 2). These relationships also showed a highly significant linear fit, indicating that mean concentrations of FPG increase with the shift of Spd + Spm from low to high quartiles. Similarly, there was a significant positive linear trend between increasing fructosamine concentration and increasing Spd + Spm levels in Model 1, although this trend was not significant in Model $2(P$ for trend $=0.081$ ). The positive linear trend between increasing HOMA-IR with increasing Spd + Spm concentrations approached the significance level $(P$ for trend $=0.057$ and 0.073 in Model 1 and Model 2, respectively), although neither model revealed any apparent differences in means of HOMA-IR. Conversely, the concentration of Spm seemed to totally lack association with any relevant glycemic indices in terms of both the difference in adjusted means and linear trend.

\section{Discussion}

The relationship between the whole blood polyamine concentration and metabolic risk factors among healthy populations has to date not been subjected to epidemiological assessment, presumably because of the unavailability of simple and inexpensive polyamine quantification methods validated against such sophisticated, precise ones as HPLC. However, in a previous study we confirmed that the colorimetric assay, as applied in this study, produces polyamine values that correlate well with those obtained by HPLC [10], and concluded that this assay method is a simple, highly sensitive, and practical one for clinical use. Since this study required the determination of the polyamine concentration of a large number of blood samples, the strong points this colorimetric assay - convenience and validity - were great assets. Although our original method used packed erythrocytes for $\mathrm{Spd}+\mathrm{Spm}$ determination, in this study we assayed the whole blood samples instead of packed ones because the samples we used had been stored in a deep freezer and thawed immediately prior to the assay. This methodological constraint required post-measurement correction to account for the plasma volume because polyamines found in the blood plasma are extremely low [23]. Although such a correction could preferably be made using hematocrit (Hct), we calculated the polyamine concentration per $\mathrm{Hb}$ for correction purposes due to the absence of Hct measurements for our subjects. However, we assumed that our correction resulted in indices highly proportional to the Hct-corrected ones given the high correlation between $\mathrm{Hct}$ and $\mathrm{Hb}(r=0.93)$, an assumption which has been confirmed in more recent data from 4034 
Table 1 Basic characteristics of 622 men according to quartiled Spm + Spd concentrations ${ }^{\mathrm{a}}$

\begin{tabular}{|c|c|c|c|c|c|}
\hline \multirow[t]{2}{*}{ Basic characteristics of study cohort ${ }^{\mathrm{b}}$} & \multicolumn{4}{|c|}{ Quartile of Spd + Spm concentration (range) ${ }^{b}$} & \multirow[t]{2}{*}{$P$ for effect } \\
\hline & $\begin{array}{l}\mathrm{Q} 1(\leq 0.082) \\
(n=155)\end{array}$ & $\begin{array}{l}\mathrm{Q} 2(\leq 0.102) \\
(n=156)\end{array}$ & $\begin{array}{l}\mathrm{Q} 3(\leq 0.121) \\
(n=156)\end{array}$ & $\begin{array}{l}\mathrm{Q} 4(>0.121) \\
(n=155)\end{array}$ & \\
\hline Age (years) & $49.5 \pm 6.6$ & $50.0 \pm 6.3$ & $51.7 \pm 6.0$ & $51.3 \pm 6.1$ & 0.004 \\
\hline Body mass index $\left(\mathrm{kg} / \mathrm{m}^{2}\right)$ & $23.0 \pm 2.4$ & $23.3 \pm 2.6$ & $23.5 \pm 2.6$ & $23.7 \pm 2.6$ & 0.119 \\
\hline Fasting plasma glucose (mmol/l) & $5.62 \pm 1.13$ & $5.54 \pm 0.75$ & $5.93 \pm 1.21$ & $5.97 \pm 1.67$ & 0.003 \\
\hline Fasting plasma insulin $(\mu \mathrm{U} / \mathrm{ml})$ & $5.35 \pm 2.86$ & $5.89 \pm 6.15$ & $6.06 \pm 3.28$ & $6.45 \pm 5.99$ & 0.240 \\
\hline HOMA-IR ${ }^{\mathrm{d}}$ & $1.34 \pm 0.74$ & $1.45 \pm 1.36$ & $1.62 \pm 0.99$ & $1.69 \pm 1.58$ & 0.042 \\
\hline Fructosamine $(\mu \mathrm{mol} / \mathrm{l})$ & $251 \pm 34$ & $255 \pm 26$ & $257 \pm 38$ & $260 \pm 47$ & 0.145 \\
\hline \multicolumn{6}{|l|}{ Polyamine-to-hemoglobin ratio $(\mu \mathrm{mol} / \mathrm{g})$} \\
\hline $\mathrm{Spd}+\mathrm{Spm}$ & $0.060 \pm 0.023$ & $0.092 \pm 0.005$ & $0.110 \pm 0.006$ & $0.158 \pm 0.093$ & $<0.001$ \\
\hline Spm & $0.034 \pm 0.022$ & $0.042 \pm 0.018$ & $0.053 \pm 0.019$ & $0.078 \pm 0.060$ & $<0.001$ \\
\hline \multicolumn{6}{|l|}{ Smoking status (\% of subjects) } \\
\hline Never & 35.5 & 35.9 & 35.9 & 32.3 & \multirow[t]{3}{*}{0.882} \\
\hline Past & 19.4 & 21.8 & 24.4 & 25.2 & \\
\hline Current & 45.2 & 42.3 & 39.7 & 42.6 & \\
\hline \multicolumn{6}{|l|}{ Drinking status (\% of subjects) } \\
\hline None & 27.1 & 27.6 & 29.5 & 34.2 & \multirow[t]{4}{*}{0.094} \\
\hline Light & 20.6 & 27.6 & 23.1 & 21.3 & \\
\hline Moderate & 20.6 & 26.9 & 16.7 & 19.4 & \\
\hline Heavy & 31.6 & 17.9 & 30.8 & 25.2 & \\
\hline \multicolumn{6}{|l|}{ Leisure-time physical activity (\% of subjects) } \\
\hline Not very active & 79.2 & 76.5 & 76.8 & 78.6 & \multirow[t]{3}{*}{0.575} \\
\hline Somewhat active & 13.0 & 12.4 & 16.8 & 15.6 & \\
\hline Regularly active & 7.8 & 11.1 & 6.5 & 5.8 & \\
\hline \multicolumn{6}{|c|}{ Present history of chronic disease (\% of subjects) } \\
\hline Yes & 14.8 & 16.0 & 21.2 & 18.1 & 0.481 \\
\hline
\end{tabular}

Spd, Spermidine; Spm, spermine

${ }^{\text {a }}$ Quartiled Spm + Spd concentrations are represented by polyamine-to-hemoglobin ratio

b Values in the upper part of the table only are given as means \pm standard deviation

c $P$ value by one-factor analysis of variance for continuous variables, and chi-square test for categorical variables

${ }^{\mathrm{d}}$ Homeostasis model assessment-insulin resistance

e Any disease under treatment, including hypertension, diabetes, dyslipidemia, stroke, heart disease, or hyperuricemia

male local government workers available from the health check-up program of 2000.

The uncorrected concentrations of polyamines determined in this study appear to be close to those obtained in our previous study [25], although direct comparison requires that differences in the characteristics of the subjects, polyamine quantification method, and correction method for polyamine concentrations be taken into consideration. Saeki et al. demonstrated that the Spd and Spm levels of packed erythrocytes were $14.1 \pm 3.1$ and $8.4 \pm 2.8 \mathrm{nmol} / \mathrm{ml}$, respectively, in 27 healthy persons and that these had significantly increased to 164.0 and $111.0 \mathrm{nmol} / \mathrm{ml}$ in patients with malignant neoplasm [26]. A similar elevation of urinary polyamines was also observed in a comparison of patients with a solid tumor and normal, healthy individuals $(2.7 \pm 0.54$ vs. $1.2 \pm 0.16 \mathrm{mg} \mathrm{Spd} / \mathrm{g}$ creatinine, and $0.6 \pm 0.28$ vs. $0.04 \pm 0.007 \mathrm{mg}$ of $\mathrm{Spm} / \mathrm{g}$ creatinine) [2]. In addition, a post-mortem study of the human frontal cortex contents of $\mathrm{Spd}$ and $\mathrm{Spm}$ in elderly patients with Down syndrome $(99.7 \pm 20.0$ and $38.6 \pm 2.1 \mathrm{nmol} / \mathrm{g}$ wwt, respectively) and Alzheimer disease $(93.5 \pm 18.2$ and $32.2 \pm 3.7 \mathrm{nmol} / \mathrm{g}$ wwt, respectively) were significantly lower than those in control subjects $(186.1 \pm 34.7 \mathrm{nmol}$ and $52.8 \pm 2.7 \mathrm{nmol} / \mathrm{g} w w t$, respectively) [27].

Because of their polycationic nature, polyamines are known to bind to negatively charged macromolecules, such as nucleic acid [3]. However, the biological significance that erythrocytes, despite lacking DNA/RNA and polyamine biosynthetic activity, carry the majority of 
Table 2 Basic characteristics of 622 men according to quartiled Spm concentrations ${ }^{\mathrm{a}}$

\begin{tabular}{|c|c|c|c|c|c|}
\hline \multirow[t]{2}{*}{ Basic characteristics of study cohort ${ }^{\mathrm{b}}$} & \multicolumn{4}{|c|}{ Quartile of Spm concentration (range) } & \multirow[t]{2}{*}{$P$ for effect ${ }^{\mathrm{C}}$} \\
\hline & $\begin{array}{l}\mathrm{Q} 1(\leq 0.033) \\
(n=155)\end{array}$ & $\begin{array}{l}\mathrm{Q} 2(\leq 0.046) \\
(n=156)\end{array}$ & $\begin{array}{l}\mathrm{Q} 3(\leq 0.063) \\
(n=156)\end{array}$ & $\begin{array}{l}\mathrm{Q} 4(>0.063) \\
(n=155)\end{array}$ & \\
\hline Age (years) & $49.3 \pm 6.3$ & $50.8 \pm 6.7$ & $51.4 \pm 6.1$ & $51.0 \pm 6.0$ & 0.015 \\
\hline Body mass index $\left(\mathrm{kg} / \mathrm{m}^{2}\right)$ & $23.1 \pm 2.6$ & $23.2 \pm 2.6$ & $23.7 \pm 2.6$ & $23.5 \pm 2.5$ & 0.201 \\
\hline Fasting plasma glucose $(\mathrm{mmol} / \mathrm{l})$ & $5.58 \pm 1.23$ & $5.76 \pm 1.20$ & $5.85 \pm 1.33$ & $5.85 \pm 1.21$ & 0.195 \\
\hline Fasting plasma insulin $(\mu \mathrm{U} / \mathrm{ml})$ & $5.57 \pm 3.03$ & $5.51 \pm 2.94$ & $6.54 \pm 6.40$ & $6.14 \pm 5.80$ & 0.186 \\
\hline HOMA-IR ${ }^{\mathrm{d}}$ & $1.39 \pm 0.80$ & $1.43 \pm 0.86$ & $1.67 \pm 1.45$ & $1.60 \pm 1.55$ & 0.119 \\
\hline Fructosamine $(\mu \mathrm{mol} / \mathrm{l})$ & $257 \pm 37$ & $253 \pm 31$ & $257 \pm 39$ & $256 \pm 40$ & 0.732 \\
\hline \multicolumn{6}{|l|}{ Polyamine-to-hemoglobin ratio $(\mu \mathrm{mol} / \mathrm{g})$} \\
\hline Spd + Spm & $0.76 \pm 0.32$ & $0.94 \pm 0.24$ & $1.16 \pm 0.81$ & $1.35 \pm 0.63$ & $<0.001$ \\
\hline Spm & $0.23 \pm 0.11$ & $0.39 \pm 0.04$ & $0.54 \pm 0.05$ & $0.91 \pm 0.56$ & $<0.001$ \\
\hline \multicolumn{6}{|l|}{ Smoking status (\% of subjects) } \\
\hline Never & 32.3 & 39.7 & 35.3 & 32.3 & \multirow[t]{3}{*}{0.831} \\
\hline Past & 22.6 & 20.5 & 23.1 & 24.5 & \\
\hline Current & 45.2 & 39.7 & 41.7 & 43.2 & \\
\hline \multicolumn{6}{|l|}{ Drinking status (\% of subjects) } \\
\hline None & 25.8 & 26.3 & 35.9 & 30.3 & \multirow[t]{4}{*}{0.061} \\
\hline Light & 28.4 & 26.9 & 14.1 & 23.2 & \\
\hline Moderate & 22.6 & 16.7 & 25.0 & 19.4 & \\
\hline Moderate & 23.2 & 30.1 & 25.0 & 27.1 & \\
\hline \multicolumn{6}{|c|}{ Leisure-time physical activity (\% of subjects) } \\
\hline Not very active & 81.6 & 77.3 & 75.0 & 77.0 & \multirow[t]{3}{*}{0.726} \\
\hline Somewhat active & 10.4 & 14.3 & 16.7 & 16.4 & \\
\hline Regularly active & 7.8 & 8.4 & 8.3 & 6.6 & \\
\hline \multicolumn{6}{|c|}{ Present history of chronic disease (\% of subjects $)^{\mathrm{e}}$} \\
\hline Yes & 14.8 & 19.9 & 15.4 & 20.0 & 0.472 \\
\hline
\end{tabular}

Spm, Spermine

${ }^{\text {a }}$ Quartiled Spm + Spd concentrations are represented by polyamine-to-hemoglobin ratio

b Values in the upper part of the table only are given as means \pm standard deviation

c $P$ value by one-factor analysis of variance for continuous variables, and chi-square test for categorical variables

${ }^{\mathrm{d}}$ Homeostasis model assessment-insulin resistance

e Any disease under treatment, including hypertension, diabetes, dyslipidemia, stroke, heart disease, or hyperuricemia

circulating polyamines by actively concentrating polyamines via their transporters [28] has been poorly understood. In this respect, one hypothesis recently under intensive investigation is that polyamines accumulated in erythrocytes function as quenching glycating agents. A number in vitro experiments have demonstrated that polyamines have a potent antiglycation effect [29, 30]. Although glycated hemoglobin (HbA1c) is also a widely used marker of glycation, in addition to fructosamine, we did not include HbA1c in the statistical model because HbAlc was measured only in a portion of the study population. However, we did confirm that a significant relationship does exist between both $\mathrm{Spd}+\mathrm{Spm}$ and $\mathrm{Spm}$ with $\mathrm{HbA1c}$ by conducting a similar multivariate analysis of the data set collected from a population in a rural area (unpublished data).

The most salient result of our study is the highly significant difference in mean FPG concentration across the quartiles of the Spd + Spm. Moreover, the increase in FPG concentration showed a significant linear fit increasing levels of Spd + Spm. This result supports the possibility of a feedback mechanism for protection against glycation under the hyperglycemic condition; i.e., the elevation in FPG levels under physiological conditions results in the elevation of Spd + Spm concentrations to subdue the glycation reaction. Since the formation of pyrraline, one of the major AGEs, occurs in a manner dose-dependent on the glucose concentration [30], it is likely that the amount of 
Table 3 Multivariate-adjusted mean of fasting glucose, insulin, HOMA-IR, and fructosamine levels according to quartiled Spm + Spd and Spm concentrations $^{\mathrm{a}}$

\begin{tabular}{|c|c|c|c|c|c|c|}
\hline \multirow[t]{2}{*}{ Basic characteristics of study cohort } & \multicolumn{4}{|c|}{ Quartile of Spd + Spm concentration } & \multirow[t]{2}{*}{$P$ for effect ${ }^{\mathrm{b}}$} & \multirow[t]{2}{*}{$P$ for trend ${ }^{\mathrm{C}}$} \\
\hline & $\mathrm{Q} 1(n=155)$ & $\mathrm{Q} 2(n=156)$ & $\mathrm{Q} 3(n=156)$ & $\mathrm{Q} 4(n=155)$ & & \\
\hline \multicolumn{7}{|l|}{ Fasting plasma glucose $(\mathrm{mmol} / \mathrm{l})$} \\
\hline Model $1^{\mathrm{d}}$ & 5.67 & 5.56 & 5.89 & 5.94 & 0.019 & 0.010 \\
\hline Model $2^{\mathrm{e}}$ & 5.96 & 5.84 & 6.18 & 6.25 & 0.013 & 0.007 \\
\hline \multicolumn{7}{|l|}{ Fasting plasma insulin $(\mu \mathrm{U} / \mathrm{ml})$} \\
\hline Model 1 & 5.60 & 5.95 & 5.98 & 6.21 & 0.685 & 0.246 \\
\hline Model 2 & 5.88 & 6.11 & 6.15 & 6.40 & 0.784 & 0.320 \\
\hline \multicolumn{7}{|l|}{ HOMA-IR } \\
\hline Model 1 & 1.41 & 1.47 & 1.60 & 1.62 & 0.282 & 0.057 \\
\hline Model 2 & 1.52 & 1.55 & 1.68 & 1.72 & 0.329 & 0.073 \\
\hline \multicolumn{7}{|l|}{ Fructosamine $(\mu \mathrm{mol} / \mathrm{l})$} \\
\hline Model 1 & 251 & 256 & 256 & 260 & 0.213 & 0.045 \\
\hline \multirow[t]{3}{*}{ Model 2} & 261 & 264 & 264 & 268 & 0.315 & 0.081 \\
\hline & \multicolumn{4}{|c|}{ Quartile of Spm concentration } & \multirow[t]{2}{*}{$P$ for effect $^{\mathrm{b}}$} & \multirow[t]{2}{*}{$P$ for trend } \\
\hline & $\mathrm{Q} 1(n=155)$ & $\mathrm{Q} 2(n=156)$ & $\mathrm{Q} 3(n=156)$ & $\mathrm{Q} 4(n=155)$ & & \\
\hline \multicolumn{7}{|l|}{ Fasting plasma glucose $(\mathrm{mmol} / \mathrm{l})$} \\
\hline Model $1^{\mathrm{d}}$ & 5.64 & 5.76 & 5.82 & 5.84 & 0.493 & 0.141 \\
\hline Model $2^{\mathrm{e}}$ & 5.92 & 6.02 & 6.14 & 6.12 & 0.380 & 0.102 \\
\hline \multicolumn{7}{|l|}{ Fasting plasma insulin $(\mu \mathrm{U} / \mathrm{ml})$} \\
\hline Model 1 & 5.79 & 5.62 & 6.31 & 6.07 & 0.513 & 0.304 \\
\hline Model 2 & 5.95 & 5.80 & 6.57 & 6.24 & 0.443 & 0.308 \\
\hline \multicolumn{7}{|l|}{ HOMA-IR } \\
\hline Model 1 & 1.45 & 1.46 & 1.61 & 1.59 & 0.453 & 0.158 \\
\hline Model 2 & 1.53 & 1.54 & 1.72 & 1.67 & 0.338 & 0.137 \\
\hline \multicolumn{7}{|l|}{ Fructosamine $(\mu \mathrm{mol} / \mathrm{l})$} \\
\hline Model 1 & 258 & 253 & 257 & 256 & 0.647 & 0.952 \\
\hline Model 2 & 267 & 261 & 266 & 264 & 0.428 & 0.815 \\
\hline
\end{tabular}

HOMA-IR, Homeostasis model assessment-insulin resistance; Spd, spermidine; Spm, spermine

${ }^{\text {a }}$ Quartiled Spm + Spd and Spm concentrations represented by polyamine-to-hemoglobin ratio

b $P$ value by analysis of covariance

c Test for trend was performed using linear contrast on the assumption of equally-spaced quartiles from low to high

d Adjusted for age and BMI in Model 1

e Adjusted for age, BMI, smoking status, drinking habit, leisure-time physical activity, and present history of chronic disease in Model 2

circulating polyamines required to inhibit the Maillard reaction is proportional to the concentration of FPG. The linear relationship between the Spd $+\mathrm{Spm}$ concentrations and fructosamine can be explained in the same manner as fructosamine can be regarded as an integrated glycemic index due to its high correlation with the glycated total proteins [21]. The lack of an apparent relationship between $\mathrm{Spm}$ and the glycemic profiles was unexpected because previous reports had revealed a potent glycation-inhibitory effect of AGE production by Spd compared to that of Spm or putrescine [30]. In the biosynthetic pathway of polyamines, the less inhibitory Spd is thought to be readily catalyzed into the more potent $\mathrm{Spm}$ to function as an antiglycating agent in response to the hyperglycemic conditions, and $\mathrm{Spm}$ is believed to be readily catabolized back to Spd. As such, the Spd + Spm concentration may be a more sensitive biomarker to reflect the antiglycation effect of circulating polyamines. However, these explanations are mere speculation in the absence of relevant studies demonstrating any similarities and dissimilarities in the behavior of circulating polyamines in in vitro versus in vivo experiments. 
Any causal interpretations of the relationship between circulating polyamines and glycemic profiles illustrated here should be considered to be tentative, given certain limitations of our study. Since polyamine metabolism has been acknowledged to involve a number of regulatory elements in the elaborate biosynthesis/degradation/uptake pathways [3], the observed relationship between the polyamine concentrations and glycemic profiles may not be straightforward, but subject to the homeostatic control of some - perhaps as yet unknown - important intervening factors. Such factors include ornithine decarboxylase, which is the rate-limiting key enzyme of polyamine biosynthesis, and/or the cellular transport system, which remains poorly characterized in mammals [31, 32]. Although the observed association had some implications for the significance of polyamine measurements in the general populations, cross-sectional analyses like the one reported here are inevitably susceptible to bias, which is being properly handled in a prospective cohort study currently underway for the follow-up of our study subjects until glucose impairment and other metabolic disorders occur.

In summary, based on these results, which were unexpected in light of previous in vitro experiments, we hypothesize that the levels of circulating polyamines are kept raised under hyperglycemic conditions to quench the glycation reaction in healthy individuals, reflecting the existence of a feedback mechanism that protects against the adverse effects of reactive carbonyl compounds.

Acknowledgements We gratefully acknowledge the financial assistance for the present study in the form of a Grant-in-Aid for Scientific Research from the Ministry of Education, Culture, Sports, Science and Technology of Japan (No. 17390184).

\section{References}

1. Vlassara H, Palace MR. Diabetes and advanced glycation endproducts. J Intern Med. 2002;251:87-101.

2. Russell DH. Clinical relevance of polyamines as biochemical markers of tumor kinetics. Clin Chem. 1977;23:22-7.

3. Gugliucci A. Polyamines as clinical laboratory tools. Clin Chim Acta. 2004;344:23-5.

4. Nakamura K, Nakazawa Y, Ienaga K. Acid-stable fluorescent advanced glycation end products: vesperlysines $\mathrm{A}, \mathrm{B}$, and $\mathrm{C}$ are formed as crosslinked products in the Maillard reaction between lysine or proteins with glucose. Biochem Biophys Res Commun. 1997;232:227-30.

5. Russell DH, Russell SD. Relative usefulness of measuring polyamines in serum, plasma, and urine as biochemical markers of cancer. Clin Chem. 1975;21:860-3.

6. Hsu HC, Thomas T, Sigal LH, Thomas TJ. Polyamine-fas interactions: inhibition of polyamine biosynthesis in MRL-lpr/lpr mice is associated with the up-regulation of fas mRNA in thymocytes. Autoimmunity. 1999;29:299-309.

7. David SA, Silverstein R, Amura CR, Kielian T, Morrison DC. Lipopolyamines: novel antiendotoxin compounds that reduce mortality in experimental sepsis caused by gram-negative bacteria. Antimicrob Agents Chemother. 1999;43:912-9.

8. Alhonen L, Parkkinen JJ, Keinanen T, Sinervirta R, Herzig KH, Janne J. Activation of polyamine catabolism in transgenic rats induces acute pancreatitis. Proc Natl Acad Sci USA. 2000;97:8290-5.

9. Teti D, Visalli M, McNair H. Analysis of polyamines as markers of (patho) physiological conditions. J Chromatogr B. 2002; 781:107-49.

10. Takagi K, Tatsumi Y, Kitaichi K, Iwase M, Shibata E, Nakao M, et al. A sensitive colorimetric assay for polyamines in erythrocytes using oat seedling polyamine oxidase. Clin Chim Acta. 2004;340:219-27.

11. Smith TA. Purification and properties of the polyamine oxidase of barley plants. Phytochemistry. 1972;11:899-910.

12. Smith TA. Polyamine oxidase from barley and oats. Phytochemistry. 1976;15:633-6.

13. Smith TA. Further properties of the polyamine oxidase from oat seedlings. Phytochemistry. 1977;16:1647-9.

14. Suzuki O, Matsumoto T, Oya M, Katsumata Y, Samejima K. A new fluorometric assay for spermidine synthase. Anal Biochem. 1981;115:72-7.

15. Matsumoto T, Furuta T, Nimura Y, Suzuki O. Increased sensitivity of the fluorometric method of Snyder and Hendley for oxidase assays. Biochem Pharmacol. 1982; 31:2207-9.

16. Smith TA. Polyamine oxidation by enzymes from Hordeum vulgare and Pisum sativum seedlings. Phytochemistry. 1974;13:1075-81.

17. Lockwood DH, East LE. Studies of the insulin-like actions of polyamines on lipid and glucose metabolism in adipose tissue cells. J Biol Chem. 1974;249:7717-22.

18. Giudicelli Y, Rebourcet MC, Nordmann R, Nordmann J. Insulinlike effect of some polyamines on lipoprotein lipase from rat adipose tissue. FEBS Lett. 1976;62:74-6.

19. Gilad VH, Halperin R, Chen-Levy Z, Gilad GM. Cyclic changes of plasma spermine concentrations in women. Life Sci. 2002;72:135-41.

20. Matsuda M, DeFronzo RA. Insulin sensitivity indices obtained from oral glucose tolerance testing: comparison with the euglycemic insulin clamp. Diabetes Care. 1999;22:1462-70.

21. Mosca A, Carenini A, Zoppi F, Carpinelli A, Banfi G, Ceriotti F, et al. Plasma protein glycation as measured by fructosamine assay. Clin Chem. 1987;33:1141-6.

22. Bergeron C, Bansard JY, Le Moine P, et al. Erythrocyte spermine levels: a prognostic parameter in childhood common acute lymphoblastic leukemia. Leukemia. 1997;11:31-6.

23. Bardocz S. The role of dietary polyamines. Eur J Clin Nutr. 1993;47:683-90.

24. Mabuchi T, Yatsuya H, Tamakoshi K, Otsuka R, Nagasawa N, Zhang $\mathrm{H}$, et al. Association between serum leptin concentration and white blood cell count in middle-aged Japanese men and women. Diabetes Metab Res Rev. 2005;21:441-7.

25. Kondo T, Hamajima N, Nishio K, Ishida Y, Imai R, Ueyama J, et al. Association of a polymorphism in the ornithine decarboxylase gene with whole blood polyamine concentrations in a non-smoking healthy population. J Health Sci. 2007;53:40612.

26. Saeki Y, Uehara N, Shirakawa S. Sensitive fluorimetric method for the determination of putrescine, spermidine and spermine by high-performance liquid chromatography and its application to human blood. J Chromatogr. 1978;145:221-9.

27. Seidl R, Beninati S, Cairns N, Singewald N, Risser D, Bavan H, Nemethova M, Lubec G. Polyamines in frontal cortex of patients with Down syndrome and Alzheimer disease. Neurosci Lett. 1996;206:193-5. 
28. Cooper KD, Shukla JB, Rennert OM. Polyamine distribution in cellular compartments of blood and in aging erythrocytes. Clin Chim Acta. 1976;73:71-8.

29. Gugliucci A, Menini T. The polyamines spermine and spermidine protect proteins from structural and functional damage by AGE precursors: a new role for old molecules? Life Sci. 2003;72:2603-16.

30. Mendez JD, Leal LI. Inhibition of in vitro pyrraline formation by L-arginine and polyamines. Biomed Pharmacother. 2004;58:598604.
31. Thomas T, Thomas TJ. Polyamines in cell growth and cell death: molecular mechanisms and therapeutic applications. Cell Mol Life Sci. 2001;58:244-58.

32. Igarashi K, Kashiwagi K. Polyamine transport in bacteria and yeast. Biochem J. 1999;344:633-42. 